\title{
Phytohemagglutinin-Induced Peripheral Blood Cytogenetics: A Valid Means for Diagnosis and Imatinib Therapy Monitoring of Chronic Phase Chronic Myeloid Leukemia Patients
}

Niyaz A Azad1, Shahid M Baba1, Zafar A Shah" ${ }^{1 *}$, Roohi Rasool', Arshad A Pandith², Shiekh A Aziz", Javed Rasool ${ }^{3}$ and Fayaz A Dar²

${ }^{1}$ Department of Immunology and Molecular Medicine, Sher-I-Kashmir Institute of Medical Sciences, Srinagar, Jammu and Kashmir, India

${ }^{2}$ Advanced Centre for Human Genetics, Sher-I-Kashmir Institute of Medical Sciences, Srinagar, Jammu and Kashmir, India

${ }^{3}$ Department of Clinical Hematology, Sher-I-Kashmir Institute of Medical Sciences, Srinagar, Jammu and Kashmir, India

${ }^{4}$ Department of Medical Oncology, Sher-I-Kashmir Institute of Medical Sciences, Srinagar, Jammu and Kashmir, India

\begin{abstract}
Background: Conventional cytogenetic studies have been viewed as the standard follow-up method for Chronic Myeloid Leukemia patients on Imatinib. However, this approach is beset with high probability of poor metaphase index. In this study, we evaluated the application of Phytohemagglutinin (PHA)-induced peripheral blood culture based cytogenetic analysis (Karyotyping) in diagnosis and Imatinib treatment monitoring of the Chronic Phase CML patients.
\end{abstract}

Methods: The patient samples were subjected to the PHA-induced peripheral blood culture based cytogenetic technique (Karyotyping) to establish their baseline cytogenetic status followed by their follow up Karyotyping twice at the end of 3 and 6 months of treatment. The simultaneous quantitative PCR (q-PCR) assay for BCR-ABL fusion gene transcript on the samples corresponding to their baseline as well as the follow up cytogenetic status was also carried out to authenticate the cytogenetic findings.

Results: Complete Cytogenetic Response (CCR) and Partial Cytogenetic Response (PCR) was initially observed in $09(30 \%)$ and $16(53.3 \%)$ respectively of $30 \mathrm{CML}$ patients with $05(16.6 \%)$ patients showing no such response at the end of 3 months. At 6 months, $25(83.3 \%)$ and $02(6.6 \%)$ showed CCR and PCR respectively with $03(10 \%)$ of patients without any response. The findings completely correlated with the hematological response, the q-PCR assay as well as the overall disease condition observed in the patients.

Conclusion: As acquiring bone-marrow sample involves morbid consequences for patients and metaphases yielded thereby are difficult to analyze, PHA-induced peripheral blood Karyotyping was explored as an alternative. It was found to have significant potential in serving as a valid tool in the diagnosis and assessment of follow up response to Imatinib mesylate treatment of patients with chronic phase CML.

Keywords: CML; Cytogenetic response; Imatinib; Peripheral blood; Karyotyping; q-PCR; PHA

\section{Background}

Patients with Chronic Myeloid Leukemia (CML) have a clonal hematopoietic disorder which is characterized by a specific translocation $\mathrm{t}(9 ; 22)(\mathrm{q} 34 ; \mathrm{q} 11.2)$ yielding the truncated Chromosome-22, called the Philadelphia chromosome that harbors an abnormal fusion or hybrid gene called BCR-ABL. This gene produces a dysregulated 'tyrosine kinase' enzyme, which signals the marrow to make too many white blood cells and is responsible for abnormal myeloproliferation in CML [1].

Chronic Myeloid Leukemia (CML) is a relatively rare disease with a worldwide annual incidence rate ranging from less than 1.0 to 2.0 per 100,000 persons. CML represents $14 \%$ of all cases of leukemia (Leukemia and Lymphoma Society, 2004). About 3\% of childhood leukemia's are CML, and $10 \%$ of cases of CML occur in children aged 5-20 years [2]. Chronic Myeloid Leukemia (CML) is occurring in younger age group in our region compared to the median age at the time of presentation being 53 years as reported in western literature [1]. In India, CML was seen prevalent in third and fourth decades [3]. As per the study conducted by Arshad et al. [4], the leukemia's rank fifth in order of occurrence among the cancers prevalent in Kashmir valley (North India), wherein CML figures as the second most incident leukemia after Acute Lymphoid Leukemia (ALL). The male to female ratio in our series was 1.14:1 which is comparable with the studies from other parts of the world $[5,6]$.
Philadelphia chromosome $(\mathrm{Ph})$ is the cytogenetic hallmark found in $95 \%$ of patients with chronic myeloid leukemia (CML) $[7,8]$. It is the karyotypic detection of this very cytogenetic marker that is instrumental in establishing the diagnosis as well as helps monitor treatment response in CML patients. In the formation of the $\mathrm{Ph}$ translocation, two fusion genes are generated: BCR-ABL on the $\mathrm{Ph}$ chromosome and ABL-BCR on the chromosome 9 participating in the reciprocal translocation $\mathrm{t}(9 ; 22)(\mathrm{q} 34 ; \mathrm{q} 11)$ [9]. The BCR-ABL gene encodes a protein with dysregulated (uncontrolled) tyrosine kinase activity, which triggers an abnormal proliferation of the myeloid lineage of the white cell component of blood [10]. The presence of this protein in CML cells is strong evidence of its disease-causing role. The efficacy in CML of a drug-Tyrosine kinase inhibitor- that inhibits the BCR-ABL

${ }^{*}$ Corresponding author: Zafar Amin Shah, Professor and Head, Department of Immunology and Molecular Medicine, Sher-I-Kashmir Institute of Medical Sciences Srinagar - 190011, Jammu and Kashmir, India, Tel: 0194-2401013 (2262); E-mail: azad.niyaz6@gmail.com

Received May 29, 2015; Accepted July 17, 2015; Published July 21, 2015

Citation: Azad NA, Baba SM, Shah ZA, Rasool R, Pandith AA, Aziz SA, et al. (2015) Phytohemagglutinin-Induced Peripheral Blood Cytogenetics: A Valid Means for Diagnosis and Imatinib Therapy Monitoring of Chronic Phase Chronic Myeloid Leukemia Patients. J Cancer Sci Ther 7: 242-248. doi:10.4172/1948-5956.1000356

Copyright: @ 2015 Azad NA, et al. This is an open-access article distributed under the terms of the Creative Commons Attribution License, which permits unrestricted use, distribution, and reproduction in any medium, provided the original author and source are credited. 
Citation: Azad NA, Baba SM, Shah ZA, Rasool R, Pandith AA, Aziz SA, et al. (2015) Phytohemagglutinin-Induced Peripheral Blood Cytogenetics: A Valid Means for Diagnosis and Imatinib Therapy Monitoring of Chronic Phase Chronic Myeloid Leukemia Patients. J Cancer Sci Ther 7: 242-248. doi:10.4172/1948-5956.1000356

tyrosine kinase has provided the final proof that the BCR-ABL oncoprotein is the principal cause of CML.

Clinically, CML progresses through three distinct phases, which are broadly classified on the basis of the number of the blast cells present into 'chronic phase' with up to $15 \%$ of blasts; 'accelerated phase' with $15 \%$ to $30 \%$ of blasts and 'blast crisis' with $>30 \%$ of blasts present [1].

There is no single standard therapy for patients with CML. Advances in bone marrow transplantation and the effects of recombinant interferon-alpha combined with chemotherapy with either hydroxyurea or cytarabine have proven clinically important. The recent introduction of Imatinib represents a new treatment option [11-13]. Imatinib is a molecularly targeted therapy that inhibits the oncogenic fusion protein BCR-ABL, which is a dysregulated Tyrosine kinase enzyme. It has demonstrated efficacy in the treatment of CML as a 'tyrosine kinase inhibitor', particularly in the chronic phase. Several studies indicate the prognostic value of the major as well as complete cytogenetic response and have correlated the acquisition of additional cytogenetic abnormalities with Imatinib resistance during treatment $[14,15]$.

As CML is among the considerably incident leukemias and ranks as the fifth largest category of cancer burden in the Kashmir valley (North India) [4], we aimed at evaluating the efficacy of the said peripheral blood cytogenetic technique, which is first of its kind in our region, in detecting the disease and monitoring the cytogenetic response in CML patients treated with tyrosine kinase inhibitor (Imatinib mesylate).

\section{Methods}

\section{Patients and samples}

A total of 30 patients in the chronic phase of CML were recruited from the Departments of Medical Oncology, Sher-i-Kashmir Institute of Medical Sciences (SKIMS) and Clinical Hematology, SKIMS and included in the study after a thorough approval from the 'Institute Ethics Committee' (IEC) of SKIMS and subjected to prospective evaluation of their response to the Imatinib treatment at the Department of Immunology \& Molecular Medicine, SKIMS from October 2013 to November 2014. The diagnosis of CML was based on characteristic peripheral blood smear and bone marrow examination findings which were confirmed by presence of Philadelphia chromosome on peripheral blood cytogenetic studies as well as detection of BCR-ABL translocation by q-PCR. All the patients received $400 \mathrm{mg}$ daily of Imatinib mesylate for the first three months and subsequently underwent cytogenetic evaluation of their peripheral blood. This was followed by continuing the same treatment for the next 3 months with dose enhancement of up to $800 \mathrm{mg}$ twice daily only in those patients who did not show any cytogenetic response after the first 3 months.

\section{Cytogenetic analysis}

Chromosomal analysis was performed before Imatinib therapy and twice three-monthly after the treatment as per the routine cytogenetic analysis [16] with the modification of adding Phytohemagglutinin (PHA-Gibco)-40 $\mu \mathrm{l} / 5 \mathrm{ml}$ of media at culture initiation. Peripheral blood karyotypes were obtained from such cultures along with a tube each of unstimulated PHA-free culture for comparative purposes as $1 \mathrm{ml}$ of Peripheral blood was inoculated in $5 \mathrm{ml}$. of RPMI 1640 (Cell Clone) culture media with $10 \%$ fetal calf serum (Gibco) at $37^{\circ} \mathrm{C}$ for 48 72 hours. Cell cultures were treated with Colchicine (Loba Chemie- 1 $\mathrm{mg} / 10 \mathrm{ml})$ along with Ethidium bromide $(1 \mathrm{mg} / 10 \mathrm{ml})$ for the final hour of incubation. Cells were subsequently harvested by standard procedures (hypotonic shock: $0.075 \mathrm{M}$ ) and fixed in methanol-acetic acid (3:1). GTG banding was performed as described by Seabright [17], and chromosomes were identified and arranged according to the International System for Human Cytogenetic Nomenclature (ISCN) [18] with the help of a computerized work station- 'Cytovision'. The number of cells investigated for each patient at each analysis ranged from 20 to 30 metaphases.

\section{Molecular analysis (q-PCR)}

$3 \mathrm{ml}$ of freshly drawn samples were put to density gradient centrifugation (Ficoll, Sigma) to get the white cell component of the peripheral whole blood to be subjected to Trizol (Amresco) RNA extraction. The extracted RNA was checked for purity and integrity by DEPC-treated Gel electrophoresis which yielded intact and prominent bands for $28 \mathrm{~S}, 18 \mathrm{~S}$ and $5 \mathrm{~S}$ r-RNA, thereby lending an indirect but authentic confirmation about the integrity of the desired fusion m-RNA transcript. The RNA after normalization to the concentration of approximately $500 \mathrm{ng}$ was subjected to the integrated c-DNA synthesis and real-time amplification by using Taqman probe based BCR-ABL transcript quantitation kit (Geno-Sen's Genome Diagnostics Pvt. Ltd.) on the Agilent Stratagene Mx-3000-P Real-time-PCR platform.

The diagnosis of CML was based on characteristic peripheral blood smear and bone marrow examination findings, which was confirmed by the presence of Philadelphia chromosome in excess of $80 \%$ of metaphases upon PHA-induced peripheral blood cytogenetic studies and detection of BCR-ABL fusion transcripts in excess of $80 \%$ by q-PCR.

\section{Response evaluation}

The object of evaluation for cytogenetic response is the Metaphase spread or chromosome spread on the slide. The good metaphase index means adequate number and healthy looking chromosome spreads yielded per slide/sample. While, it is conventional wisdom to expect lower metaphase index in respect of the malignant metaphases yielded in a PHA-induced peripheral blood culture compared to their normal or non-malignant counterparts, we modified the response evaluation criteria followed in bone marrow cytogenetics, which is: complete cytogenetic response (CCyR: $0 \%$ of $\mathrm{Ph}+$ cells); partial response (PCyR: from $1-35 \%$ of $\mathrm{Ph}+$ cells); minor response (MrCR: $36-65 \%$ of $\mathrm{Ph}+$ cells); minimal response (MiCR: 66-95\% of $\mathrm{Ph}+$ cells) and no response (NoCR: $>95 \%$ of $\mathrm{Ph}+$ cells) with: complete cytogenetic response as: (CCR: $0 \%$ of $\mathrm{Ph}+$ cells); partial response as: (PCR: PCyR+MrCR) and no cytogenetic response as: (NCR: MiCR+NoCR). Further, the cytogenetic response was correlated with the q-PCR assay findings and other clinical evaluation like state of Spleen and TLC counts. With regard to the BCR-ABL transcript load, three quantification ranges of '0-40\%, ' $40-60 \%$ ' and '60-80\%' were set as reference scale corresponding to CCR, PCR and NCR respectively (Tables 1 and 2).

\section{Results}

The CML patients included 16 males (53.3\%) and 14 females (46.7\%) within the age range of $18-70.11(36.7 \%)$ cases belonged to age group $>45$ years and the rest $19(63.3 \%)$ were $<45$ years of age. All the patients had raised Total Leukocyte Count (TLC) with overall mean value of $80 \times 10^{3} / \mu$ land different grades of Splenomegaly along with the q-PCR status of $>60 \%$ of BCR-ABL fusion transcript load. All the patients subjected to initial Karyotyping in order to establish their baseline cytogenetic status were found to be Philadelphia positive $(\mathrm{Ph}+)$ (Figure 1 and Table 1).

At the end of 3 months of treatment, the follow-up karyotyping 
Citation: Azad NA, Baba SM, Shah ZA, Rasool R, Pandith AA, Aziz SA, et al. (2015) Phytohemagglutinin-Induced Peripheral Blood Cytogenetics: A Valid Means for Diagnosis and Imatinib Therapy Monitoring of Chronic Phase Chronic Myeloid Leukemia Patients. J Cancer Sci Ther 7 : 242-248. doi:10.4172/1948-5956.1000356

revealed $25(83.3 \%)$ patients with a marked cytogenetic response, which is comparable with the parameter of 'Major Cytogenetic Response' (MCR) (as discussed in similar studies elsewhere), and wherein 16 of 25 (64\%) patients showed Partial Cytogenetic Response (PCR) and 09 of $25(36 \%)$ patients were found to have Complete Cytogenetic Response (CCR) with total Philadelphia negative status (Table 1). Subsequently, the 16 cases with 'PCR' also showed a clear graduation to the 'CCR' at the end of 6 months of treatment (Table 2). 05 of the total 30 (16.7\%) cases returned with their complete Philadelphia positive status and No Cytogenetic Response (NCR) at all at the end of first 3 months of treatment with one male patient reporting the occurrence of clonal evolution changes of Trisomy- 8 and Isochromosome-17q along with the Philadelphia chromosome (Figure 2). In this group who were unresponsive to the initial treatment, Imatinib dose was subsequently escalated to $800 \mathrm{mg}$ daily for further 3 months. The second follow-up karyotyping of these patients revealed response improvement in 02 of 05 cases $(40 \%)$ that graduated to the partial cytogenetic response from no cytogenetic response, whereas the rest 03 of 05 (60\%) cases, including the clonal evolution one, showed no such improvement and retained their Philadelphia positive cytogenetic status. A notable finding of the study was that there was a remarkable response improvement at 6 months rather than 3 months of treatment duration with CCR as well as molecular response at 6 months being more significant than at 3 months $(p<0.0007)$ (Table 3, Figure 4).

Besides, the treatment response didn't show any gender bias as males and females seemed almost equally responsive in terms of 'CCR', 'PCR', and 'NCR' with 05,09 and 02 of the total 16 males constituting 'CCR', 'PCR' and 'NCR' respectively and 04,07 and 03 of the total 14 females constituting 'CCR', 'PCR' and 'NCR' respectively at the end of

\begin{tabular}{|c|c|c|c|c|c|}
\hline \multicolumn{2}{|c|}{ Particulars } & \multirow{3}{*}{$\begin{array}{c}\text { Number } \\
16\end{array}$} & \multicolumn{3}{|c|}{ Cytogenetic Response (Peripheral Blood) } \\
\hline & & & Complete & Partial & No response \\
\hline \multirow[t]{2}{*}{ Gender } & Male & & 05 & 09 & 02 \\
\hline & Female & 14 & 04 & 07 & 03 \\
\hline \multirow[t]{2}{*}{ Age } & $>45$ & 11 & 04 & 06 & 01 \\
\hline & $<45$ & 19 & 05 & 10 & 04 \\
\hline \multirow[t]{2}{*}{ Dwelling } & Urban & 12 & 03 & 06 & 03 \\
\hline & Rural & 18 & 06 & 10 & 02 \\
\hline \multirow[t]{2}{*}{ TLC+Spleen (Baseline) } & Raised & 30 & ------ & ------ & ------ \\
\hline & Normal & 00 & ------ & ------ & ------ \\
\hline \multirow[t]{2}{*}{ BCR-ABL q-PCR value (Baseline) } & $>80 \%$ & 30 & ------ & ------ & ------ \\
\hline & $<80 \%$ & 00 & ------ & ------ & ------ \\
\hline \multirow[t]{2}{*}{ Karyotyping (Baseline) } & $\mathrm{Ph}+\mathrm{ve}(>80 \%)$ & 30 & ------ & ------ & ------ \\
\hline & Ph-ve & 00 & ----- & ----- & ----- \\
\hline \multirow[t]{2}{*}{ TLC+Spleen (Follow up) } & Raised & 05 & 00 & 00 & 05 \\
\hline & Normal & 25 & 09 & 16 & 00 \\
\hline \multirow[t]{3}{*}{ BCR-ABL $\quad$ q-PCR value (Follow-up) } & \multicolumn{2}{|c|}{$0-40 \%$} & 09 & ------ & ------ \\
\hline & \multicolumn{2}{|c|}{$40-60 \%$} & ------ & 16 & ----- \\
\hline & \multicolumn{2}{|c|}{$60-80 \%$} & ------ & ------ & 05 \\
\hline \multirow[t]{2}{*}{ Karyotyping (Follow up) } & $\mathrm{Ph}+\mathrm{ve}$ & 05 & 00 & 00 & 05 \\
\hline & Ph-ve & 25 & 09 & 16 & 00 \\
\hline
\end{tabular}

Table 1: Patients particulars and 'Peripheral blood cytogenetic response' at 3 months of 'Imatinib' treatment.

\begin{tabular}{|c|c|c|c|c|c|}
\hline \multicolumn{2}{|c|}{ Particulars } & \multirow{3}{*}{$\begin{array}{c}\text { Number } \\
16\end{array}$} & \multicolumn{3}{|c|}{ Cytogenetic Response (Peripheral Blood) } \\
\hline & & & Complete & Partial & No response \\
\hline \multirow[t]{2}{*}{ Gender } & Male & & 14 & 01 & 01 \\
\hline & Female & 14 & 11 & 01 & 02 \\
\hline \multirow[t]{2}{*}{ Age } & $>45$ & 11 & 10 & 00 & 01 \\
\hline & $<45$ & 19 & 15 & 02 & 02 \\
\hline \multirow[t]{2}{*}{ Dwelling } & Urban & 12 & 09 & 01 & 02 \\
\hline & Rural & 18 & 16 & 01 & 01 \\
\hline \multirow[t]{2}{*}{ TLC+Spleen (Baseline) } & Raised & 30 & --.--- & -.---- & -.--- \\
\hline & Normal & 30 & ----- & ----- & ----- \\
\hline \multirow[t]{2}{*}{ BCR-ABL $\quad q-P C R$ value (Baseline) } & $>80 \%$ & 30 & ----- & $-\mathbf{- o -}^{---}$ & $-\mathbf{- o -}^{-}$ \\
\hline & $<80 \%$ & 00 & --.--- & -.---- & -.--- \\
\hline \multirow[t]{2}{*}{ Karyotyping (Baseline) } & $\mathrm{Ph}+\mathrm{ve}(>80 \%)$ & 30 & ------ & ----- & ----- \\
\hline & Ph-ve & 00 & ------ & ----- & $-\mathbf{- o -}^{--}$ \\
\hline \multirow[t]{2}{*}{ TLC+Spleen (Follow up) } & Raised & 03 & 00 & 00 & 03 \\
\hline & Normal & 27 & 25 & 02 & 00 \\
\hline \multirow[t]{3}{*}{ BCR-ABL $\quad$ q-PCR value (Follow-up) } & \multicolumn{2}{|c|}{$0-40 \%$} & 25 & ----- & $-\mathbf{- o -}^{--}$ \\
\hline & \multicolumn{2}{|c|}{$40-60 \%$} & ----- & 02 & ----- \\
\hline & & ----- & $-\mathbf{- o -}^{-}$ & 03 \\
\hline \multirow[t]{2}{*}{ Karyotyping (Follow up) } & \multicolumn{2}{|c|}{$60-80 \%$} & 00 & 00 & 03 \\
\hline & \multicolumn{2}{|c|}{\begin{tabular}{l|l} 
Ph-ve & 27
\end{tabular}} & 25 & 02 & 00 \\
\hline
\end{tabular}

Table 2: Patients particulars and 'Peripheral blood cytogenetic response' at 6 months of 'Imatinib' treatment. 


\section{y

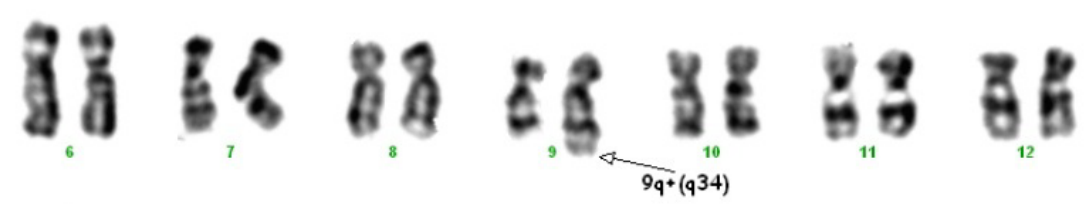

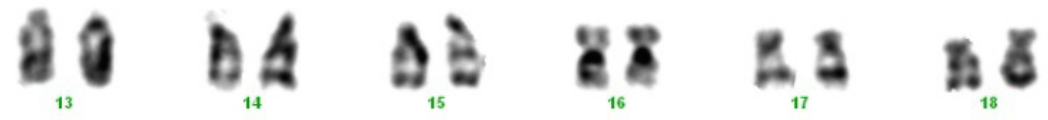

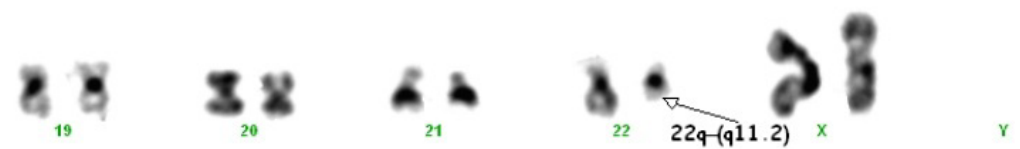

Figure 1: Representative karyotype of the Philadelphia positive chromosome complement from PHA-induced/stimulated peripheral blood culture.

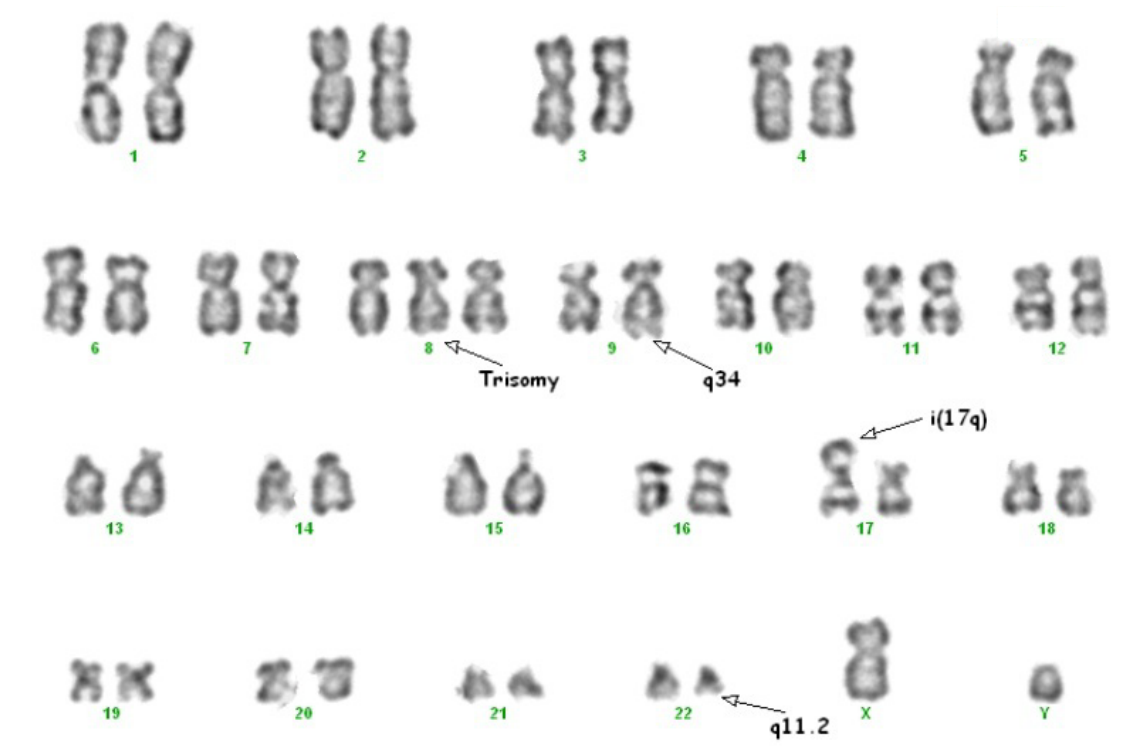

Figure 2: Representative karyotype of the clonal evolution positive chromosome complement from PHA-induced/stimulated peripheral blood culture.

\begin{tabular}{|c|c|c|c|c|}
\hline \multirow{2}{*}{ Method } & $\begin{array}{c}\text { Duration of } \\
\text { treatment }\end{array}$ & $\begin{array}{c}\text { Complete response (CCR)- 0\% } \\
\mathbf{P h +}\end{array}$ & $\begin{array}{c}\text { Partial response (PCR)-01\%- } \\
\mathbf{6 5 \%} \mathbf{P h +}\end{array}$ & $\begin{array}{c}\text { No response (NCR)-66\% ->95\% } \\
\mathbf{P h +}\end{array}$ \\
\hline \multirow{2}{*}{ Cytogenetics } & 03 months & $09(30 \%)$ & $16(53.3 \%)$ & $05(16.7 \%)$ \\
\hline & 06 months & $25(83.3 \%)$ & $02(6.7 \%)$ & $03(10 \%)$ \\
\hline BCR-ABL q-PCR value & & $0-40 \%$ & $40-60 \%$ & $60-80 \%$ \\
\hline & 03 months & $09(30 \%)$ & $16(53.3 \%)$ & $05(16.7 \%)$ \\
\hline & 06 months & $25(83.3 \%)$ & $02(6.7 \%)$ & $03(10 \%)$ \\
\hline
\end{tabular}

Table 3: Comparative cytogenetic and molecular responses at 3 and 6 months.

first 3 months of treatment. This trend continued at the end of 6 months of further treatment when 14, 01 and 01 of the total 16 males constituted 'CCR', 'PCR' and 'NCR' respectively and 11, 01 and 02 of the total 14 females constituted 'CCR', 'PCR' and 'NCR' respectively, wherein 01 each case among the males and females of the initial 'NCR' group ( 05 of total 30 patients) graduated to 'PCR' from 'NCR' after dose escalation of
$800 \mathrm{mg}$. The 03 of 05 cases retained their 'NCR' status even after the said dose escalation and included 02 females and 01 male. Simultaneously the same series of samples evaluated for molecular response showed a $100 \%$ concordance with the cytogenetic response (Table 3, Figures 4 and 5). 


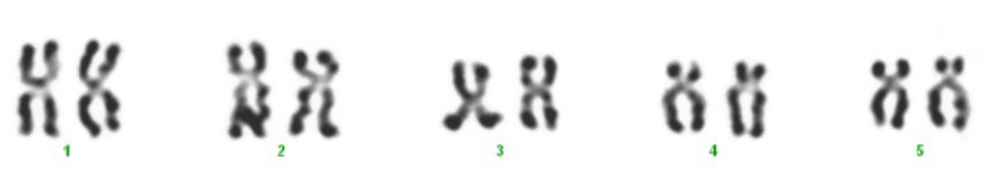 \\ й вй

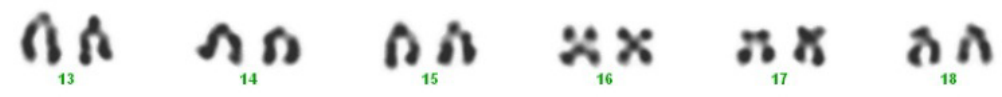

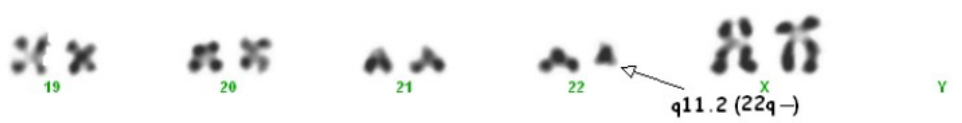

Figure 3: Representative karyotype of the Philadelphia positive chromosome complement from PHA-uninduced/unstimulated peripheral blood culture.

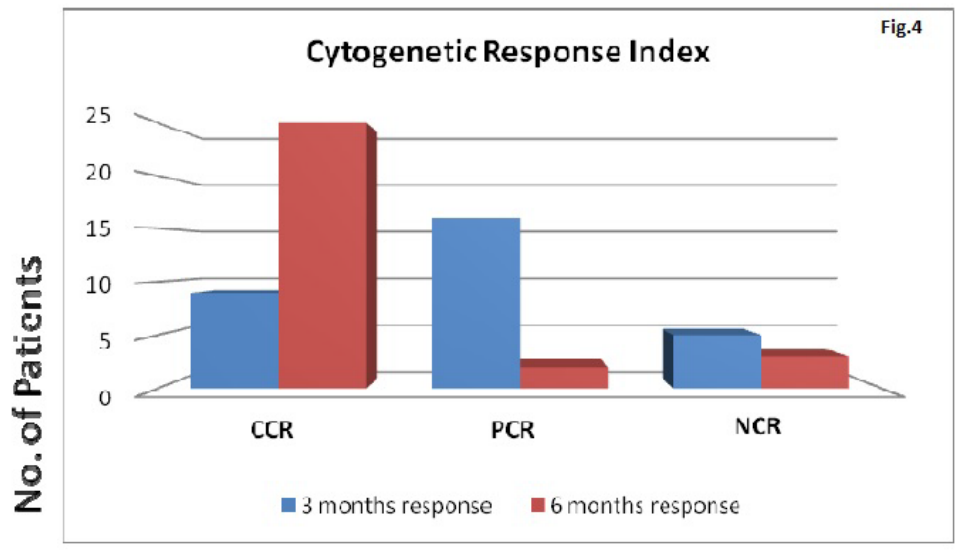

CCR: Complete Cytogenetic Response

PCR: Partial Cytogenetic Response

NCR: NO Cytogenetic Response

Figure 4: Representative of differential cytogenetic response at 3 and 6 months duration.

All the results were interpreted and correlated accordingly as per the Karyotypes obtained from PHA-induced peripheral blood cultures (Figure 1), which were clearly better at detection of the marker chromosomal aberrations than the non-PHA cultures (Figure 3).

\section{Discussion}

Conventional cytogenetics requires a bone marrow sample and evaluation of 20 or more metaphases for the Philadelphia chromosome $(\mathrm{Ph}+)$. Categories of cytogenetic response include minimal cytogenetic response, with $36 \%$ to $95 \% \mathrm{Ph}+$ metaphases; partial cytogenetic response, with $1 \%$ to $35 \% \mathrm{Ph}+$ metaphases; major cytogenetic response, with $0 \%$ to $35 \% \mathrm{Ph}+$ metaphases; and complete cytogenetic response, with $0 \% \mathrm{Ph}+$ metaphases. Although cytogenetic studies are associated with a wide confidence interval as the number of metaphases evaluated is limited, the association between cytogenetic response and the treatment outcomes is quite well established $[19,20]$.
Detection of $\mathrm{Ph}$ chromosome by conventional karyotyping is still considered the basis for monitoring the targeted therapy using Imatinib in CML. Monitoring the percentage of $\mathrm{Ph}$ positive cells is the best validated system for assessment of the response to tyrosine kinase inhibitors, since the cytogenetic response is the best surrogate marker of survival $[21,22]$.

Many a review literature, like Hagop Kantarjian et al. [23], on comparative analysis of monitoring tools available in the assessment of treatment response of CML patients on Imatinib acknowledge the consideration of conventional cytogenetic studies using chromosome Giemsa banding (Karyotyping) as the gold standard of treatment monitoring in view of its better performance in terms of sensitivity, least false positivity and detection of other chromosomal abnormalities in addition to the detection of the Philadelphia chromosome.

Although the conventional cytogenetic studies on bone marrow have been viewed as the standard follow-up method for patients on Imatinib, 


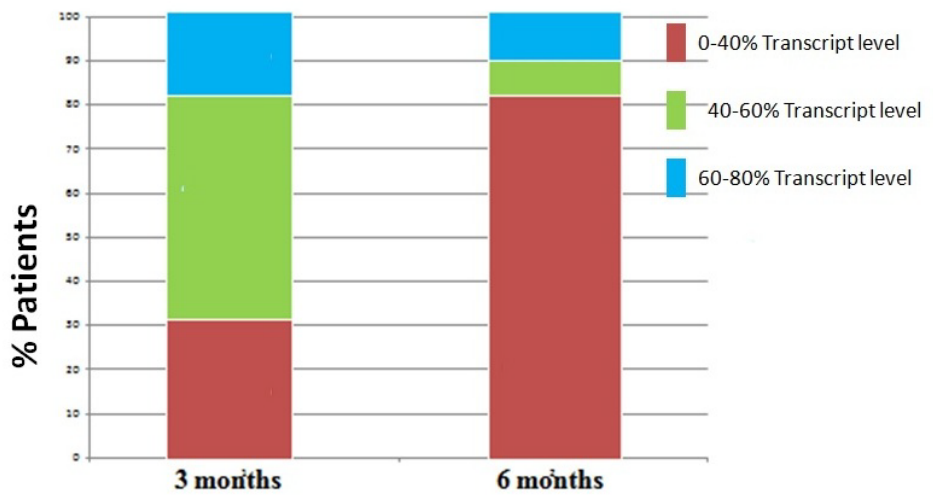

Figure 5: Representative of corresponding molecular response at 3 and 6 months of treatment.

the technique continues to be confronted with the issue of quality of the signal metaphases produced, which are compact and dysmorphic, thereby making them not amenable to prominent G-banding and hence easy and convenient analysis [24]. Accordingly, this study was taken up to evaluate the application of Phytohemagglutinin (PHA)-induced peripheral blood culture based cytogenetic analysis (Karyotyping) in diagnosis and Imatinib treatment monitoring of the Chronic Phase CML patients of Kashmir region (North India). Furthermore, we resorted to quantitative molecular analysis (q-PCR) to authenticate the cytogenetic response as it correlates well with the cytogenetic assessment as a 2 -log reduction in transcripts (to $1 \%$ from baseline) broadly equates with a CCyR, and a $1-\log$ reduction (to $10 \%$ from baseline) equating to a MCyR [25].

In our study, we found that adding PHA to this technique and doing it on the peripheral blood showed good promise of serving as a reliable alternative to the conventional PHA-free cytogenetics as there was a marked improvement in terms of the quality of the metaphases generated with PHA-induced cultures of peripheral blood (Figures 1 and 3 ). Even though the number of metaphases with marker translocation might be lesser than in conventional bone marrow cytogenetics, the PHA-induced peripheral blood metaphases, however, yielded healthy and prominently spread out chromosomes, which made the analysis less taxing and dependable [24].

It is important to note that our primary objective has been to emphasize the utility of the technique used by us for cytogenetics rather than the comprehensive evaluation of Imatinib treatment. Accordingly, we restricted the follow-up duration to 3 and 6 months only as that was enough to yield a fair idea about the working of the technique under evaluation. This is also in consonance with the European LeukemiaNet recommendations of 2013 for the management of chronic myeloid leukemia, which recommends the follow-up cytogenetics at 3,6 and 12 months as a valid response assessment option to follow Imatinib treatment. It endorses that Partial Cytogenetic Response (PCyR) at 3 months and Complete Cytogenetic Response (CCyR) from 6 months onward define optimal response, whereas no response (Philadelphia chromosome-positive cells $>95 \%$ ) at 3 months, less than PCyR at 6 months, and less than CCyR from 12 months onward define failure [26], which further vindicates the follow-up duration modality adopted by us. Besides, Hagop M. Kantarjian et al. [27] reported that those receiving Imatinib mesylate had higher incidences of complete and major cytogenetic responses at 3 months, 6 months and 9 months compared to those on different Interferon regimens and recorded Major cytogenetic response- $\mathrm{MCyR}(\mathrm{CCyR}+\mathrm{PCyR})$ in $90 \%$ and $\mathrm{CCyR}$ in $72 \%$ of patients at the median follow-up of 9 months, which is in considerable agreement with our report of MCR in $90 \%$ and CCR in $83.3 \%$ of patients at the follow-up of 6 months.

The second follow-up karyotyping of the patients (05 of 30) who didn't initially show any cytogenetic response and were subjected to dose escalation of $800 \mathrm{mg}$ of daily Imatinib manifested response improvement in 02 of 05 cases (40\%) that graduated to the partial cytogenetic response from no cytogenetic response, while as the rest 03 of 05 (60\%) cases retained their Philadelphia positive status. Similar results were also found in yet another study conducted by Kantarjian et al. where partial cytogenetic response was observed in 2 of the 20 resistant cases and the rest cases did not respond to dose escalation [28]. Such a scenario could be attributed to the phenomenon of Imatinib resistance, which may be the BCR-ABL independent type or the BCR-ABL dependent type or a combination of both. The response improvement in 02 of 05 cases upon dose escalation could be explained as a case of BCR-ABL independent Imatinib resistance, wherein the enhanced dosage of the drug has probably taken care of the interindividual variability in pharmacokinetics and dynamics of the drug e.g. the role of drug transporters (ABCB1-ABCG2) is one of specific causes of Imatinib resistance, since it can be effluxed through MDR1 (ABCB1) transporters [29]. In addition multiple mechanisms of resistance have been implicated in Imatinib mesylate therapy; these include clonal evolution, point mutations, and overexpression and amplification of $\mathrm{Bcr} / \mathrm{Abl}$. Similarly, the lack of treatment response in case of the 03 of the 05 cases could well be the case of BCR-ABL dependent Imatinib resistance e.g. the Kinase domain mutations in the BCR-ABL fusion gene are implicated in approximately $50 \%$ of Imatinib-resistant patients [30], which are refractory to even dose escalations of Imatinib. Further, we did not find any significant difference in molecular or cytogenetic response to Imatinib treatment with respect to gender or age.

\section{Conclusion}

The PHA-induced/stimulated peripheral blood cytogenetic analysis correlated remarkably well with the clinical status of the patients concerned, thereby exhibiting its significant role as a valid means for the diagnosis as well as the periodic follow-up monitoring of patients on Imatinib treatment.

\section{Acknowledgment}

We acknowledge with warm thanks the cooperation of the patients of this study as well as the resident staff of the Departments of Medical Oncology and Clinical Hematology of SKIMS for their help and support. We also thank the SKIMS administration for funding this study. 
Citation: Azad NA, Baba SM, Shah ZA, Rasool R, Pandith AA, Aziz SA, et al. (2015) Phytohemagglutinin-Induced Peripheral Blood Cytogenetics: A Valid Means for Diagnosis and Imatinib Therapy Monitoring of Chronic Phase Chronic Myeloid Leukemia Patients. J Cancer Sci Ther 7 : 242-248. doi:10.4172/1948-5956.1000356

\section{Competing interests}

The authors of this article have no competing interests.

\section{References}

1. Cortes $\mathrm{J}$ (2004) Natural history and staging of chronic myelogenous leukemia. Hematol Oncol Clin North Am 18: 569-584, viii.

2. Simons CM, Stratton CW, Kim AS (2011) Peripheral blood eosinophilia as a clue to the diagnosis of an occult Coccidioides infection. Hum Pathol 42: 449-453.

3. Deshmukh C, Saikia T, Bakshi A, Amare-Kadam P, Baisane C, et al. (2005) Imatinib mesylate in chronic myeloid leukemia: a prospective, single arm, nonrandomized study. J Assoc Physicians India 53: 291-295.

4. Pandith AA, Siddiqi MA (2012) Burden of cancers in the valley of Kashmir: 5 year epidemiological study reveals a different scenario. Tumour Biol 33 $1629-1637$.

5. Kantarjian HM, Sawyer C, Hochhaus A, Guilhot F, Schiffer C, et al. (2002) Hematologic and cytogenetic response to imatinib mesylate in chronic myelogenous leukemia. New Engl J Med 346: 645-652.

6. Crossman LC, O'Brien SG (2004) Imatinib therapy in chronic myeloid leukemia. Hematol Oncol Clin North Am 18: 605-617, viii.

7. Dewald GW, Juneau AL, Schad CR, Tefferi A (1997) Cytogenetic and molecular genetic methods for diagnosis and treatment response in chronic granulocytic leukemia. Cancer Genet Cytogenet 94: 59-66.

8. Groffen J, Heisterkamp N (1987) The BCR/ABL hybrid gene. Baillieres Clin Haematol 1: 983-999.

9. Bumm T, Muller C, Al-Ali HK, Krohn K, Shepherd P, et al. (2003) Emergence of clonal cytogenetic abnormalities in $\mathrm{Ph}$ - cells in some CML patients in cytogenetic remission to imatinib but restoration of polyclonal hematopoiesis in the majority. Blood 101: 1941-1949.

10. Rowley JD (1973) Letter: A new consistent chromosomal abnormality in chronic myelogenous leukaemia identified by quinacrine fluorescence and Giemsa staining. Nature 243: 290-293.

11. Alvarez RH, Kantarjian H, Cortes JE (2007) The biology of chronic myelogenous leukemia: implications for imatinib therapy. Semin Hematol 44: S4-14.

12. Ilaria RL Jr (2004) Animal models of chronic myelogenous leukemia. Hemato Oncol Clin North Am 18: 525-543, vii.

13. Druker BJ (2009) Perspectives on the development of imatinib and the future of cancer research. Nat Med 15: 1149-1152.

14. Braziel RM, Launder TM, Druker BJ, Olson SB, Magenis RE, et al. (2002) Hematopathologic and cytogenetic findings in imatinib mesylate-treated chronic myelogenous leukemia patients: 14 months' experience. Blood 100: 435-441.

15. Otero L, Ornellas MH, de Azevedo AM, Dobbin J, Abdelhay E, et al. (2007) The Impact of Additional Chromosomal Abnormalities in Response to Imatinib Mesylate Therapy for Chronic Myeloid Leukemia. Revista Brasileira de Cancerologia 53: 405-410.

16. Bonifazi F, deVivo A, Rosti G, Guilhot F, Guilhot J, et al. (2001) Chronic myeloid leukemia and interferon-alpha: a study of complete cytogenetic responders. Blood 98: 3074-3081.

17. Seabright M (1971) A rapid banding technique for human chromosomes. Lancet 2: 971-972.

18. Mitelman F (1995) ISCN 1995: An International System for Human Cytogenetic Nomenclature. S Karger, Basel

19. Kantarjian HM, O'Brien S, Cortes JE, Shan J, Giles FJ, et al. (2003) Complete cytogenetic and molecular responses to interferon-alpha-based therapy for chronic myelogenous leukemia are associated with excellent long-term prognosis. Cancer 97: 1033-1041.

20. Kantarjian HM, Smith TL, O'Brien S, Beran M, Pierce S, et al. (1995) Prolonged survival in chronic myelogenous leukemia after cytogenetic response to interferon-alpha therapy. The Leukemia Service. Ann Intern Med 122: 254-261.

21. O’Brien SG, Guilhot F, Larson RA, Gathmann I, Baccarani M, et al. (2003) Imatinib compared with interferon and low-dose cytarabine for newly diagnosed chronic-phase chronic myeloid leukemia. N Eng J Med 348: 994-1004.

22. Goldman JM (2007) How I treat chronic myeloid leukemia in the imatinib era Blood 110: 2828-2837

23. Kantarjian H, Schiffer C, Jones D, Cortes J (2008) Monitoring the response and course of chronic myeloid leukemia in the modern era of BCR-ABL tyrosine kinase inhibitors: practical advice on the use and interpretation of monitoring methods. Blood 111: 1774-1780.

24. Sachdeva MUS, Verma N, Rana KS, Verma S (2012) Philadelphia chromosome detection in chronic myeloid leukemia. Philadelphia chromosome detection in chronic myeloid leukemia: Utility of Phytohemagglutinin-stimulated peripheral blood culture. Indian J Pathology and Microbiol 55: 196-201.

25. Branford S, Rudzki Z, Parkinson I, Grigg A, Taylor K, et al (2004) Real-time quantitative $\mathrm{PCR}$ analysis can be used as a primary screen to identify patients with $C M L$ treated with imatinib who have BCR-ABL kinase domain mutations Blood 104: 2926-2932.

26. Baccarani M, Deininger MW, Rosti G, Hochhaus A, Soverini S, et al. (2013) European Leukemia-Net recommendations for the management of chronic myeloid leukemia: 2013. Blood 122: 872-884.

27. Kantarjian HM, Cortes JE, O'Brien S, Giles F, Garcia-Manero G, et al. (2003) Imatinib mesylate therapy in newly diagnosed patients with Philadelphia chromosome-positive chronic myelogenous leukemia: high incidence of early complete and major cytogenetic responses. Blood 101: 97-100.

28. Kantarjian HM, Talpaz M, O’Brien S, Giles F, Garcia-Manero G, et al. (2003) Dose escalation of imatinib mesylate can overcome resistance to standarddose therapy in patients with chronic myelogenous leukemia. Blood 101: 473-475

29. Gurney H, Wong M, Balleine RL, Rivory LP, McLachlan AJ, et al. (2007) Imatinib disposition and ABCB (MDR, P-glycoprotein) genotype. Clin Pharmacol Ther 82: $33-40$.

30. Jabbour E, Kantarjian H, Jones D, Talpaz M, Bekele N, (2006) Frequency and clinical significance of BCR-ABL mutations in patients with chronic myeloid leukemia treated with imatinib mesylate. Leukemia 20: 1767-1773. 